\title{
A 1.5V Bootstrapped Pass-Transistor-Based Carry Look-Ahead Circuit Suitable for Low-Voltage CMOS VLSI
}

\author{
J. H. Lou and J. B. Kuo \\ Rm. 338, Dept. of Electrical Eng., National Taiwan University \\ Roosevelt Rd., Sec. 4, \#1, Taipei, Taiwan 106-17
}

\begin{abstract}
This paper reports a $1.5 \mathrm{~V}$ bootstrapped pass-transistor-based carry look-ahead circuit suitable for CMOS VLSI using a low supply voltage. With the bootstrapped technique, the speed performance of a 4-bit carry look-ahead circuit can be enhanced by $70 \%$ at a supply voltage of $1.5 \mathrm{~V}$ as compared to the conventional Manchester carry look-ahead circuit.
\end{abstract}

\section{INTRODUCTION}

CMOS Manchester carry look-ahead circuit as shown in Fig.1 based on pass transis tors and dynamic logic techniques [1]-[4] have been used to implement arithmetic circuit. With the pass transistor configuration, Manchester CLA circuit has the smallest transistor count among all CLA circuits including domino and other static techniques [1]-[4]. The function of the MCLA circuit is: $C_{i}=G_{i}+C_{i-1} \cdot P_{i}$, for $i=1 \sim n$, where $n$ is the bit number, $G_{i}$ and $P_{i}$ are the generate and propagate signals $\left(G_{i}=X_{i} \cdot Y_{i}, P_{i}=X_{i} \oplus Y_{i}\right)$ produced from two inputs $\left(X_{i}, Y_{i}\right)$ to the half adder. In the MCLA circuit, each bit $\overline{c a r r y}$ signal $\left(\overline{C_{i}}\right)$ is low if the generate signal $\left(G_{i}\right)$ is high or if the propagate signal $\left(P_{i}\right)$ is high and the $\overline{c a r r y}$ signal of the previous bit $\left(\overline{C_{i-1}}\right)$ is low. When the carry look-ahead chain is long, as in a 64-bit adder, the ripplecarry propagation delay becomes unacceptable due to the $\mathrm{RC}$ delay of the pass transistor [3][4]. In other words, the density advantage of theMCLA circuit is offset by the drawback in the speed. This is especially serious when the supply voltage is scaled down, which is a trend for deep-submicron VLSI. In this paper, a high-speed $1.5 \mathrm{~V}$ bootstrapped pass-transistor-based carry look-ahead circuit suitable for low-voltage CMOS VLSI is described.

\section{THE $1.5 \mathrm{~V}$ BOOTSTRAPPED}

PASS-TRANSISTOR-BASED CARRY LOOK-AHEAD CIRCUI $\mathrm{T}$

Fig.2 shows a two-stage 1.5V CMOS bootstrapped dynamic logic (BDL) circuit, which is composed of the CMOS dynamic logic circuit and the CMOS bootstrapper $c$ ircuit [5]. As shown in the figure, MPD, MND,

This work is supported under R.O.C. National Science Council Contracts \#84-2622-E002-008, 011 \& \#85-2215-E0002-024.

0-7803-3694-1/97/\$10.00 $\odot 1997$ IEEE

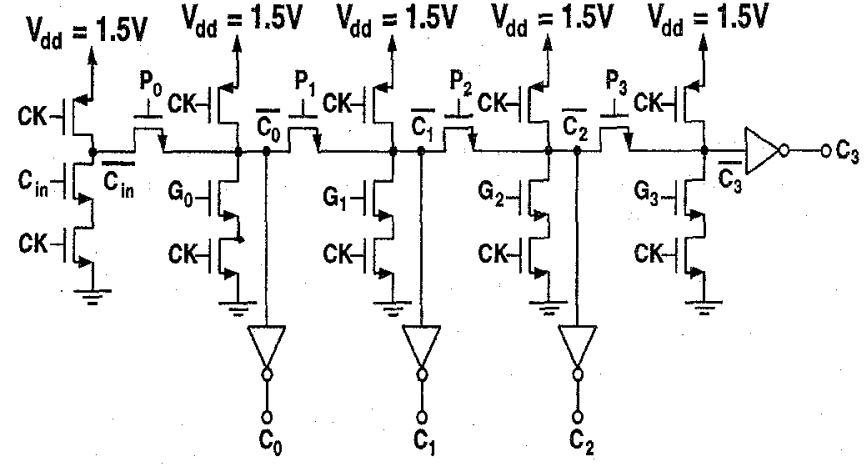

Fig. 1. A 4-bit Manchester carry look-ahead circuit.

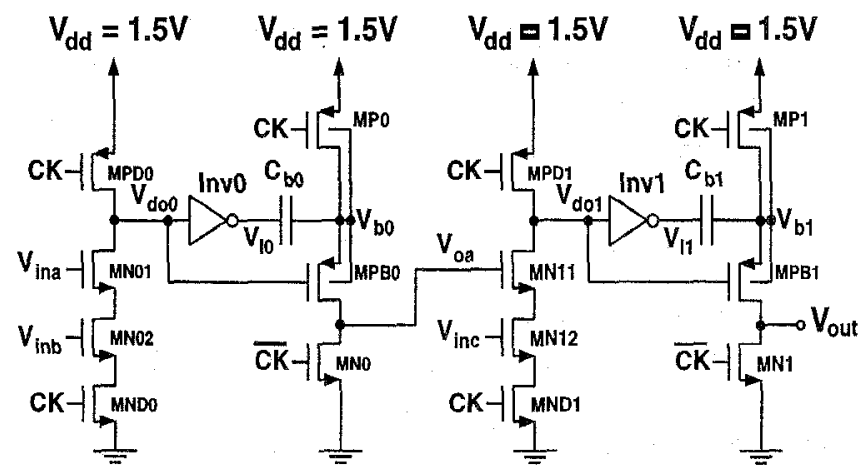

Fig. 2. The 1.5V CMOS bootstrapped dynamic logic (BDL) circuit including the CMOS bootstrapper circuit.

MN1, and MN2 comprise the dynamic logic circuit. The CMOS inverter-Inv, MP, MPB, and the bootstrap capacitor $C_{b}$ form the bootstrapper circuit. The operation of the CMOS bootstrapped dynamic logic (BDL) is like conventional domino logic, except for that the output of the CMOS bootstrapper is bootstrapped to over $V(D D)$. The function of the bootstrapper circuit will be explained in detail later.

Fig.3 shows the $1.5 \mathrm{~V}$ bootstrapped pass-transistorbased carry look-ahead circui t. As shown in the figure, the bootstrapper circuit [5], which functions as an inverter, is used to boost the input signal to the gate of the pass transistors. When the input to the bootstrapper circuit is high, MPB is off and MN is on. Therefore, the output of it is pulled low to ground. At the same time, $V_{I}$ is low, hence MP is on and $V_{b}$ is pull high to $V_{d d}$. At this time, the bootstrap transistor $M P C$, which is made of a PMOS device with its source and drain tied together, 

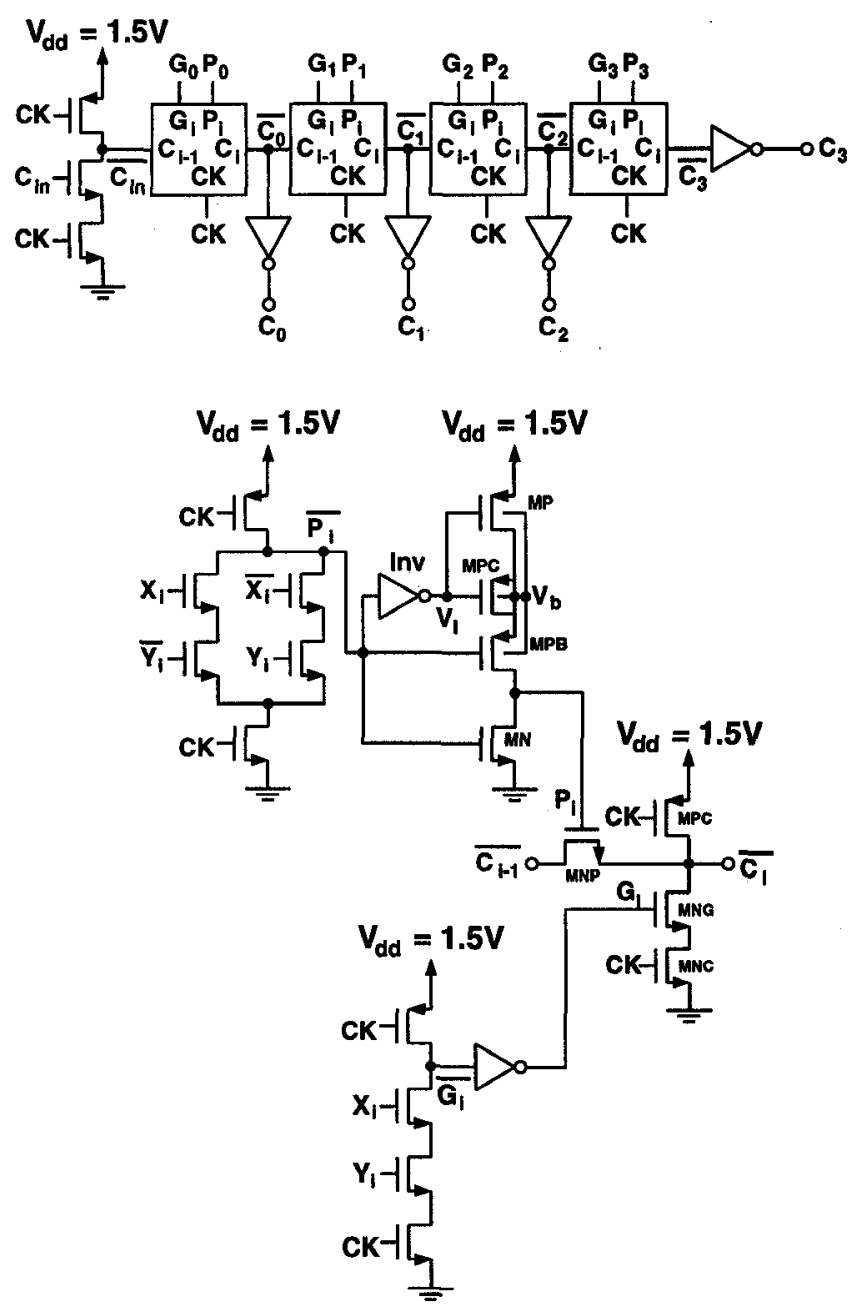

Fig. 3. (a) Block diagram of a 4-bit carry look-ahead circuit. (b) The CLA cell using $1.5 \mathrm{~V}$ bootstrapped pass-transistor-based circuit.

stores an amount of $\left(V_{d d}-\left|V_{T P}\right|\right) C_{o x} W L$ charge, where $C_{o x}$ is unit area gate oxide capacitance $\left(C_{o x}=\epsilon_{o x} / t_{o x}\right)$, and $V_{T P}$ is the threshold voltage of the PMOS device. When the input switches from high to low, MN turns off and MPB turns on. Meanwhile, $V_{I}$ changes to high and MP turns off. Since the bootstrap transistor $M P C$ turns off, the evacuated holes from the channel of the PMOS device will make $V_{b}$ go up to exceed $V_{d d}$ - the internal voltage overshoot. Consequently, the output $\left(P_{i}\right)$ will also go up to surpass $V_{d d}$. The voltage overshoot at the output of the bootstrapper circuit enhances the speed performance of the pass-transistor-based carry look-ahead circuit owing to the extra gate overdrive voltage. The advantages of the bootstrapper circuit are especially noticeable for the low supply voltage applications.

Fig.4 shows the transients of the 4-bit carry look-ahead circuit using the $1.5 \mathrm{~V}$ bootstrapped pass-transistor-based circuit and without. As shown in the figure, with the bootstrapper circuit, during the transient, the $P_{0}$ signal to gate of the pass transistor can surpass $1.5 \mathrm{~V}$. As a result, the pass transistor can turn on eariler as compared to the one in the conventional Manchester carry look-ahead
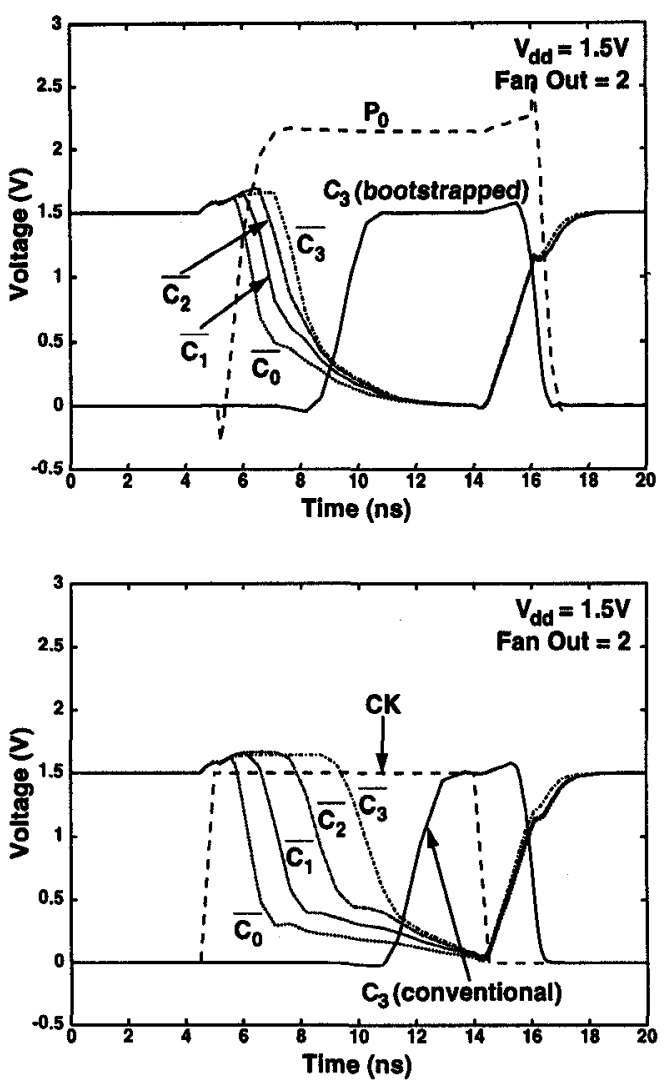

Fig. 4. Transients of the 4-bit carry look-ahead circuit (a) with and (b) without the $1.5 \mathrm{~V}$ bootstrapped pass-transistor-based circuit.

circuit. Consequently, a higher speed can be expected for the carry look-ahead circuit with the bootstrapper circuit. Fig. 5 shows the delay time versus bit number of the carry look-ahead circuit using the $1.5 \mathrm{~V}$ bootstrapped pass-transistor-based circuit and without. As shown in the figure, a consistent improvement in speed can be seen for the CLA with the bootstrapped pass-transistor-based circuit. At 8 bits, the speed enhancement is 2.1 times.

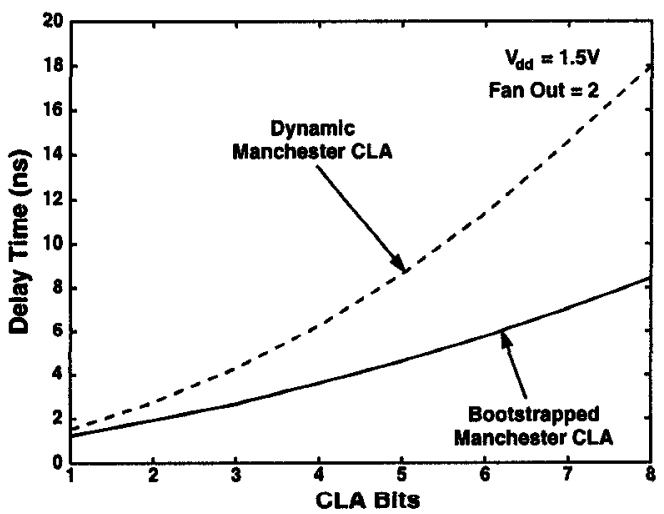

Fig. 5. Delay time versus bit number and of the carry look-ahead circuit using the $1.5 \mathrm{~V}$ bootstrapped pass-transistor-based circuit and without. 


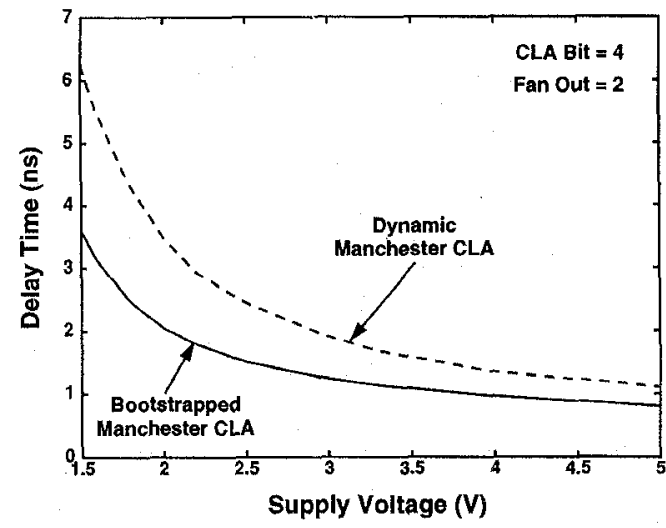

Fig. 6. Delay time versus supply voltage of the carry look-ahead circuit using the $1.5 \mathrm{~V}$ bootstrapped pass-transistor-based circuit and without.

\section{DISCUSSION}

The bootstrapped pass-transistor-based carry lookahead circuit is especially advantageous for low supply voltage applications. Fig. 6 shows the delay time versus supply voltage of the 4-bit carry look-ahead circuit using the $1.5 \mathrm{~V}$ bootstrapped pass-transistor-based circuit and without. As shown in the figure, at a supply voltage of $1.5 \mathrm{~V}$, the improvement is 1.7 times.

\section{CONCLUSION}

In this paper, a $1.5 \mathrm{~V}$ bootstrapped pass-transistorbased carry look-ahead circuit suitable for CMOS VLSI using a low supply voltage has been reported. With the bootstrapped technique, the speed performance of a 4-bit carry look-ahead circuit can be enhanced by $70 \%$ at a supply voltage of $1.5 \mathrm{~V}$ as compared to the conventional Manchester carry look-ahead circuit.

\section{REFERENCES}

[1] N. H. E. Weste and K. Eshraghian, "Principles of CMOS VLSI Design," Addison Wesley: New York, NY, 1985.

[2] J. Kernhof, M. A. Beunder, B. Hoefflinger, and W. Haas, "High-Speed CMOS Adder and Multiplier Modules for Digital Signal Processing in a Semicustom Environment," IEEE J. Solid-State Circuits, pp. 570-575, June 1989.

[3] J. B. Kuo H. J. Liao and H. P. Chen, "A BiCMOS Dynamic Carry Look-Ahead Adder Circuit for VLSI Implementation of High Speed Arithmetic Unit," IEEE J. Solid-State Circuits, pp. 375-378, Mar. 1993.

[4] J. B. Kuo, S. S. Chen, C. S. Chang, K. W. Su, and J. H. Lou, "A 1.5V BiCMOS Dynamic Logic Circuit Using a "BiPMOS Pull-Down" Structure for VLSI Inplementation of Full Adders," IEEE Trans. Circuits and Systems- I, Vol. 41, No. 4, pp. 329-332, Apr. 1994.

[5] J. H. Lou and J. B. Kuo, "A 1.5V Full-Swing Bootstrapped CMOS Large Capacitive-Load Driver Circuit Suitable for LowVoltage CMOS VLSI," IEEE J. Solid-State Circuits, Vol. 32, No. 1, pp. 119-121, Jan. 1997. 